\title{
La calidad en salud un concepto histórico vigente
}

\author{
Luz Marina Bautista-Rodriguez*
}

$\int$ 1 concepto de calidad de la atención en salud, no es diferente al que generalmente se maneja en otras disciplinas. Proveniente del latín qualitas, los diccionarios definen calidad, como el conjunto de cualidades que constituyen la manera de ser de una persona o cosa. Otros conceptos planteados por diversos autores van desde el dado a conocer por Codman en 1914, quien definió la calidad desde el punto de vista de la eficiencia afirmando que "la calidad del producto debe corresponder a la inversión que se realiza para producirla; así mismo; los productos deben tener unas características precisas"(1), lo cual requiere del establecimiento de estándares que brinden pautas de comparación interinstitucional y que permitan evaluar la calidad de los productos.

De igual forma, Donabedian en 1982 retoma la eficiencia como la maximización de los beneficios que a la vez se ve limitada por la cantidad y la calidad de los recursos otorgados, complementando toda la argumentación de su modelo estructura - proceso - resultado (2), definiendo la "calidad como la obtención del máximo beneficio para el usuario, mediante la aplicación del conocimiento y tecnología más avanzada tomando en cuenta los requerimientos del paciente, así como las capacidades y limitaciones del recurso de la institución de acuerdo con los valores sociales imperantes" (2).

Para el sector sanitario la calidad es un fenómeno multidimensional, por lo tanto, puede estar definida por diversos conceptos. La Organización Mundial de la Salud (OMS) define la calidad sanitaria como "asegurar que cada paciente reciba el conjunto de servicios, diagnósticos y terapéuticos más adecuados para conseguir una atención sanitaria optima, teniendo en cuenta los factores y los conocimientos del paciente y del servicio médico, y lograr el mejor resultado con el mínimo riesgo de efectos iatrogénicos y la máxima satisfacción del paciente en el proceso" (3).

De manera general, la definición de calidad se puede expresar en tres conceptos que son de vital importancia: el primero, que hace referencia a que la calidad es la totalidad de funciones, características o comportamientos de un bien o servicio. No hay calidad que se pueda medir por solamente la apreciación o el análisis de alguna de las partes constitutivas del servicio recibido; la calificación se hace con carácter integral, es decir, evaluando todas las características, funciones o comportamientos.

El segundo concepto, plantea que se considera un bien o un servicio con calidad cuando logra su fin, el cual es satisfacer las necesidades de los consumidores; y en este sentido, aquellos que no lo logran, no se consideran de calidad. El cliente que no sale satisfecho de un consultorio, no percibirá que fue atendido de buena manera (como a él le hubiera gustado, habiendo satisfecho sus expectativas), no habrá recibido una atención de calidad. El profesional de la salud podrá creer que trabajó calificadamente, pero si no se satisface las necesidades del paciente, éste no lo volverá a buscar por sobre todos los demás. Lógicamente que ante una menor percepción de haber sido atendida adecuadamente, la persona estará convencida que no recibió calidad, habrá menos satisfacción y más desagrado.

El tercer elemento fundamental de la definición, hace alusión a "que la calidad es un concepto subjetivo, ya que lo que para una persona es bueno, para otra inclusive, puede ser considerado como de mala calidad" (4). El grado de exigencia depende de una serie de circunstancias siendo fundamentalmente aquellas de orden cultural, social y económico. En salud, se sabe que dependiendo del nivel cultural y las expectativas del servicio las personas pueden conformarse con ser atendidos por el profesional, no importándoles en muchos casos tener que sufrir largos períodos de espera y que le sean otorgadas citas muy espaciadas, o pueden dependiendo de las circunstancias, no aceptar la prestación del servicio, si este no se caracteriza por acciones cálidas, humanas, eficaces y oportunas (4). Hoy en día, los usuarios de los servicios de salud con acceso a una mayor formación e información, son cada vez más exigentes.

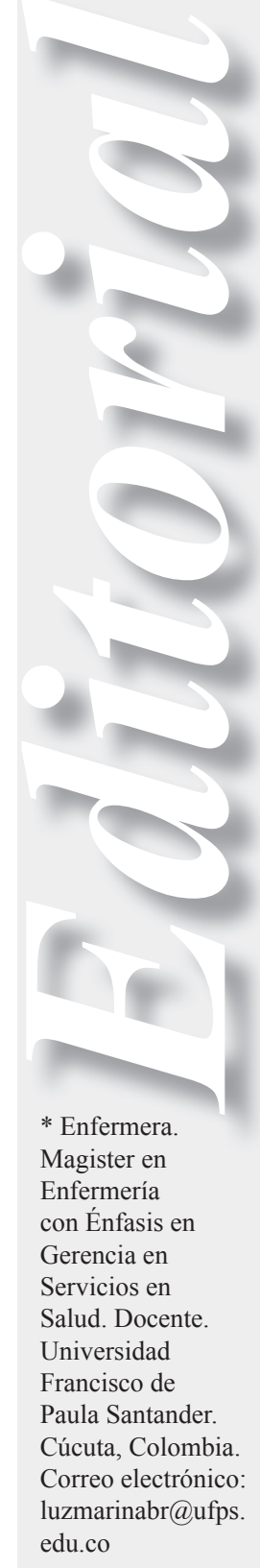


En Colombia, A partir de la expedición de la Ley 100 de 1993 y sus decretos reglamentarios se generó una gran transformación del sector salud, que hizo que las instituciones de salud asumieran retos con relación a su organización, así mismo produjo cambios en el papel ejercido por los usuarios, pues el nivel de exigencias y expectativas se modifico, haciendo que hoy en día los usuarios de los servicios de salud sean personas conscientes de sus derechos, estén informadas y requieran una atención oportuna, personalizada y eficiente lo cual implica ofertar servicios con calidad.

La calidad, se define como "el conjunto de características técnicas, científicas, materiales y humanas que debe tener la atención de salud que se provea a los beneficiarios, para alcanzar los efectos posibles con los que se obtenga el mayor numero de años de vida saludable y a un costo que sea social $\mathrm{y}$ económicamente viable para el sistema y sus afiliados" (5), entonces la calidad puede entenderse como el conjunto de atributos claves (definidos desde las expectativas de los usuarios y por las características de la competencia) presentes dentro del proceso de producción del servicio, necesarios para lograr satisfacer las necesidades de los clientes y obtener los resultados esperados.

De igual manera, el Decreto 1011 de 2006 (6) por el cual se define el sistema obligatorio de garantía de calidad del sistema general de seguridad social en salud, plantea la calidad de la atención de salud como "la provisión de servicios de salud a los usuarios individuales y colectivos de manera accesible y equitativa, a través de un nivel profesional óptimo, teniendo en cuenta el balance entre beneficios, riesgos y costos, con el propósito de lograr la adhesión y satisfacción de dichos usuarios."

Por otra parte la Organización Panamericana de la Salud (OPS) y Organización Mundial de la Salud (OMS) (7) plantean la calidad, incluyendo la seguridad del paciente, y la definen como una cualidad de la atención sanitaria esencial para la consecución de los objetivos nacionales en salud, la mejora de la salud de la población y el futuro sostenible del sistema de atención en salud.

Donabedian (8) plantea el análisis de la calidad a partir de las dimensiones de estructura, proceso y resultado, resumidos estos tres aspectos en lo técnico, lo interpersonal y las amenidades. El cuidado técnico es la aplicación de la ciencia y tecnología de la medicina y otras ciencias de la salud al manejo de un problema de salud personal. El aspecto interpersonal es el manejo de la relación social y sicológica entre el profesional y su paciente. Las amenidades se refieren a las condiciones de confort en que la atención es ofrecida.

Entonces la calidad en los servicios de salud debe dar cumplimiento a las normas técnicas que incluyen infraestructura, equipos, materiales, medicamentos, talento humano con conocimientos y habilidades para ofrecen el servicio; pero también, de las necesidades sentidas del usuario que involucran la entrega amable y respetuosa. La calidad de la atención en el proceso interpersonal es más difícil de resumir, pero debe incluir valores y normas socialmente definidas que gobiernen la interacción de los individuos en general y en situaciones particulares. Estas normas están reforzadas, en parte, por los dictados éticos de las profesiones del sector de la salud y por las expectativas de los usuarios. La relación interpersonal no se mide exclusivamente por la comunicación verbal, también, hace referencia al interés que se tiene por las personas usuarias, el cual se demuestra por la comodidad, la privacidad y por el ambiente que se les proporciona en los diferentes lugares donde se presta la atención en salud (9).

Por otra parte algunos autores (10-11) consideran que la calidad comprende otras dimensiones como: a. La dimensión técnica, concebida como la aplicación de la ciencia y la tecnología médica de un modo que proporcione los máximos beneficios para la salud sin aumentar de forma proporcional los riesgos. b. La dimensión del servicio, considera la oportunidad y continuidad en que se presta la atención, donde es importante la relación interpersonal con el paciente, las características del lugar y la facilidad de acceder al servicio. c. La dimensión de seguridad, implica obtener el máximo beneficio con el menor riesgo. d. La dimensión de costo racional, entendida como los recursos empleados para generar los beneficios sin producir riesgos.

En los servicios de salud existen dos tipos de calidad, la primera, la calidad técnica vista como la atención mediante la cual se espera poder proporcionar al cliente el máximo y más completo bienestar, después de haber tomado en cuenta el balance de ganancias y pérdidas esperadas, que acompañan el proceso de 
la atención en todas sus partes (12). La segunda, la calidad sentida constituida por la subjetividad de los usuarios, que debe ser explicada y expresada por éstos luego de la utilización de los servicios.

Este enfoque ha sido una contribución importante, pues permite medir ordenadamente las variables ligadas a la calidad de los servicios de salud, suponiendo que los resultados realmente son consecuencia de la atención proporcionada, lo cual implica que no todos los resultados puedan ser fácil y exclusivamente imputables a los procesos, y no todos los procesos dependerán directa y específicamente de la estructura.

En el caso de los servicios de salud los productos son servicios (actividades preventivas, actividades de promoción, consulta odontológica, control de enfermería etc.), estos servicios son intangibles, pero tienen un valor agregado que se convierte en la característica que identifica, diferencia y satisface. La calidad implica ir más allá del resultado final, es decir, es la forma de ofrecer el servicio desde el ingreso del usuario a la institución de salud, de esta forma surgen los momentos de verdad como herramientas primordiales para garantizar la calidad en los servicios de salud.
ISSN 1794-9831

E-ISSN 2322-7028

Vol. 13 No. 1

Ene - Jun 2016

Cúcuta, Colombia 


\section{REFERENCIAS BIBLIOGRÁFICAS}

Ene - Jun 2016

Cúcuta, Colombia
1. República de Colombia. Primera encuesta de calidad de salud percibida por los usuarios: asociación colombiana de empresas de medicina integral. Bogotá: Defensoría del Pueblo; 2001. p. 2.

2. República de Colombia. Análisis de las encuestas de calidad de vida para evaluar el impacto del nuevo sistema de seguridad social en salud en Colombia: 1993-1997. Bogotá: Departamento Nacional de Planeación, 1998. p. 45

3. Martínez-Martínez L, Avalo-Olguín M, Quintero-Crispin AL. Calidad de los servicios de enfermería: ¿utopía o realidad? Revista Desarrollo Científico en Enfermería 2001; 9(3):68-75.

4. Otero J. Qué es la calidad en salud. Lima: Ética y Marketing; 2002. p. 45.

5. Mejía B. Auditoría médica: para la garantía de la calidad. $1^{a}$ ed. Bogotá: Editorial Ltda.; 1997. p. 38.

6. República de Colombia. Ministerio de la Protección Social. Decreto 1011 de 2006, abril 3, por el cual se establece el Sistema Obligatorio de Garantía de Calidad de la Atención de Salud del Sistema General de Seguridad Social en Salud. Bogotá D.C.: El Ministerio; 2006.

7. Organización Mundial de la Salud (OMS), Organización Panamericana de la Salud (OPS). Política y estrategia regional para la garantía de la calidad de la atención sanitaria, incluyendo la seguridad del paciente. En: $27^{\mathrm{a}}$. Conferencia Sanitaria Panamericana. 59 ${ }^{a}$. Sesión del Comité Regional Washington: OPS/OMS; 2007.

8. Donabedian A. Continuidad y cambio en la búsqueda de la calidad: salud pública. México: Prensa Médica Mexicana; 1993. p. 254.

9. Escobar-Saldarriaga I, Castrillón-Agudelo MC, Pulido-Lalinde S. Calidad de atención de enfermería en salas de parto. Investigación y Educación en Enfermería 1992; X(2): p. 28.

10. Lock D, Smith D. Cómo gerenciar la calidad total: Estrategias y técnicas. Santafé de Bogotá: Legis; 1992. p. 80.

11. Gómez MA. ¿Calidad para qué? En: Organización Panamericana de la Salud (OPS), Organización Mundial de la Salud (OMS). Semana de la Calidad en Salud 2013. X Encuentro Nacional de Experiencias en Mejoramiento Continuo de la Calidad en Salud. IX Conferencia Nacional e Internacional de Calidad en Salud. Perú; OPS/ OMS; 2013.

12. Donabedian A. La calidad de la atención médica. Ediciones Científicas. México: La Prensa Mexicana; 1984. p. 7. 